Case Report

\title{
Management of esophageal perforation with fistula formation in an 80 year old woman with multiple co-morbidities
}

\author{
Jalal Almarzooq, Ayman Alkhabbaz*, Nabeel Abdulla
}

ENT Department, Salmaniya Medical Complex, Manama, Kingdom of Bahrain

Received: 03 March 2021

Accepted: 13 April 2021

\section{*Correspondence:}

Dr. Ayman Alkhabbaz,

E-mail: ayman.alkhabbaz@gmail.com

Copyright: @ the author(s), publisher and licensee Medip Academy. This is an open-access article distributed under the terms of the Creative Commons Attribution Non-Commercial License, which permits unrestricted non-commercial use, distribution, and reproduction in any medium, provided the original work is properly cited.

\begin{abstract}
Esophageal perforation due to foreign body ingestion may lead to serious complications. Here we present a case of an 80 year old lady with multiple co-morbidities who presented with a proximal esophageal perforation with fistula formation following ingestion of a fish bone. Due to her age and co-morbidities the decision was made to manage her conservatively by IV antibiotics, NG tube feeds and observation. Follow up after 2 months showed resolution of the perforation and fistula, without the patient needing any surgical intervention. This case highlights conservative management of esophageal perforation with complications in patients with co-morbidities where open surgery is not favoured.
\end{abstract}

Keywords: Esophageal perforation, Esophageal fistula, Foreign body ingestion

\section{INTRODUCTION}

Foreign body ingestion is a common clinical problem. ${ }^{1}$ Fish bones constitute one of the most commonly ingested foreign bodies and poses a risk of serious complications, including esophageal perforation and fistula formation. ${ }^{2,3}$ Management of such complications ranges from noninvasive treatment to several methods of surgical management. ${ }^{3,4}$

We report a case of fish bone ingestion which was impacted in the proximal esophagus with resultant esophageal perforation and fistula, which was accordingly managed conservatively.

\section{CASE REPORT}

The patient is an 80 year old lady, who is known to have diabetes mellitus type 2, hypertension, bronchial asthma, iron deficiency anemia and is an ex-smoker. She initially presented with history of accidental fish bone ingestion and throat pain. Neck X-ray done showed an air shadow in the upper esophagus and the patient was admitted and booked for cricopharyngoscopy and upper esophagoscopy. The following day, before the procedure, the patient refused to go to surgery and left the hospital against medical advice. Three days later she returned to hospital with increased throat pain and inability to take orally. X-ray neck was done which showed widening of the retropharyngeal space. Neck CT scan was done which showed the foreign body in the hypopharynx and proximal cervical esophagus, with a small upper esophageal perforation involving the upper third of the esophagus. Gastrograffin study was done, which showed proximal esophageal sealed perforation. She was taken to the operating room, where upper esophagoscopy was done. A large fish bone was removed from the upper esophagus and a small esophageal perforation was seen. Post-op the patient was taken to the ICU. 


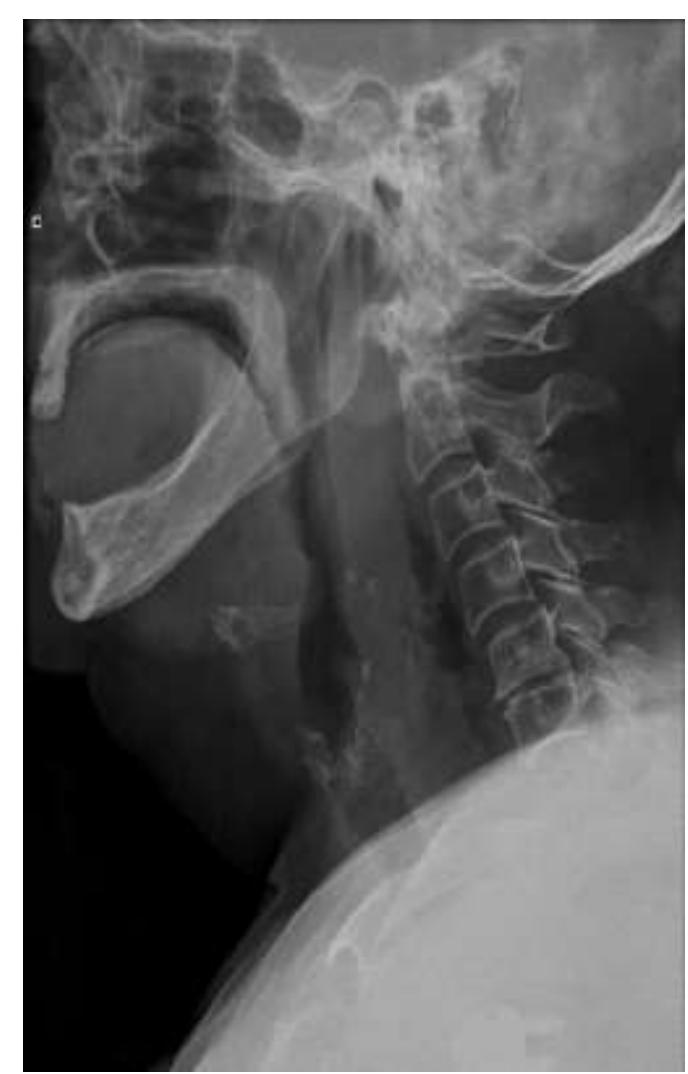

Figure 1: Lateral neck X-ray prior to removal of the foreign body showing retropharyngeal space widening.

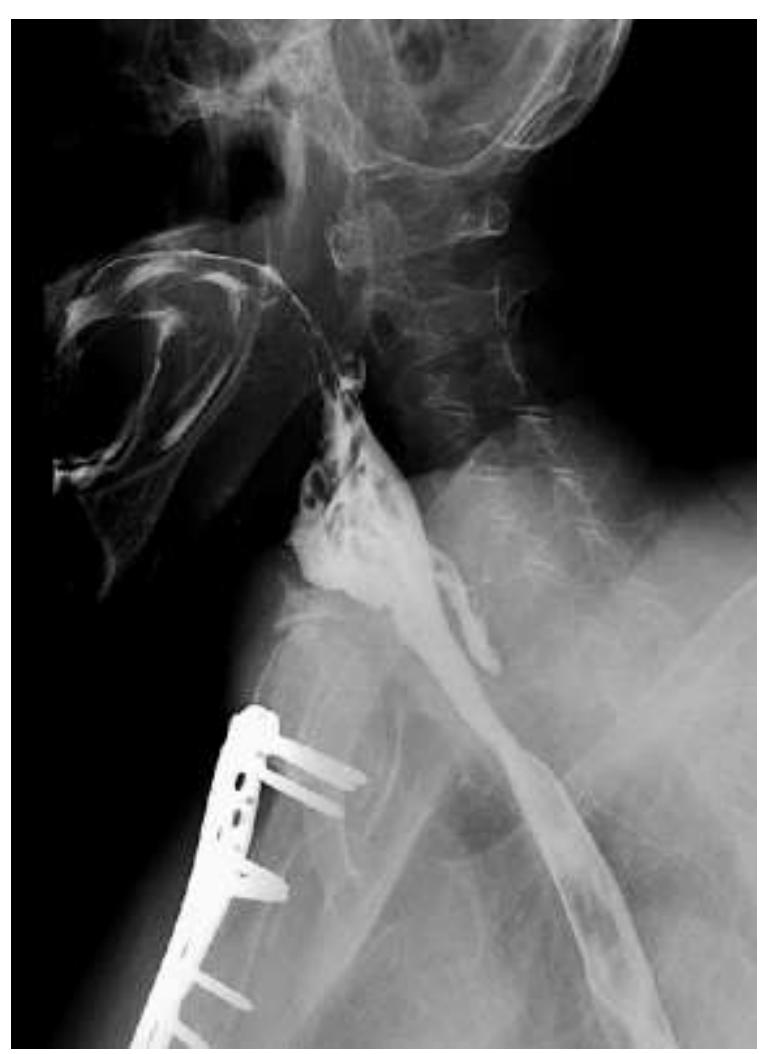

Figure 2: Barium swallow showing fistulous tract from the level of $\mathrm{C} 4$ to $\mathrm{C} 7$.

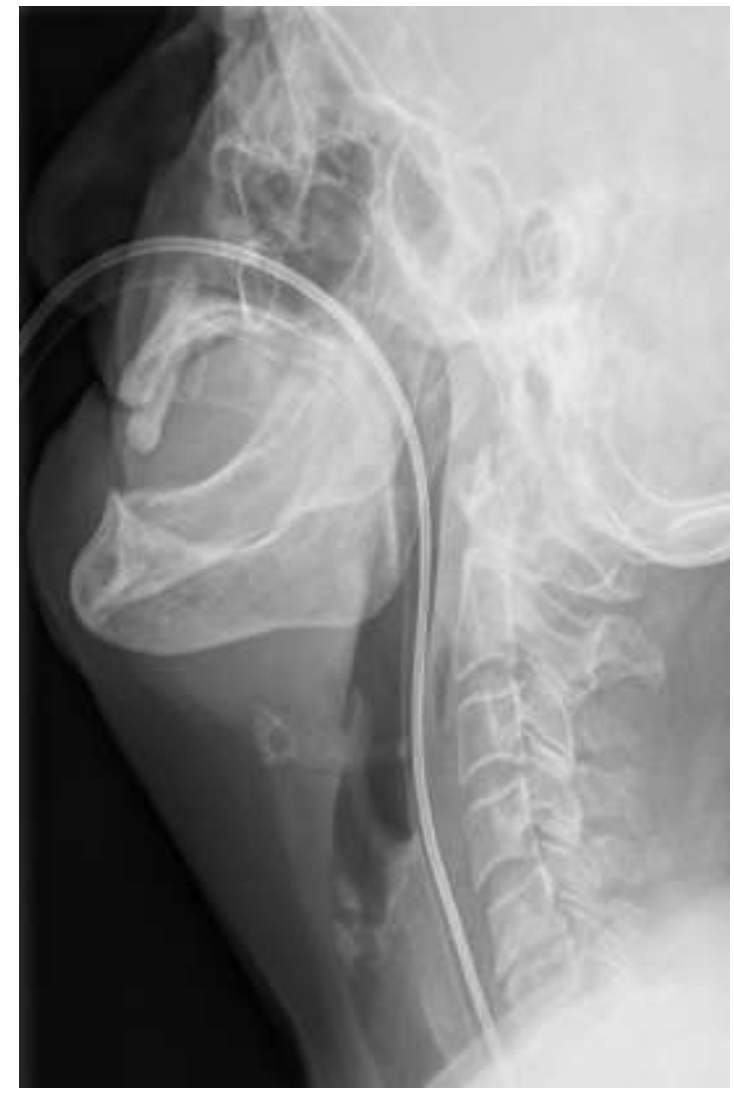

Figure 3: Lateral neck X-ray showing resolution of the retropharyngeal swelling and fistulous tract.

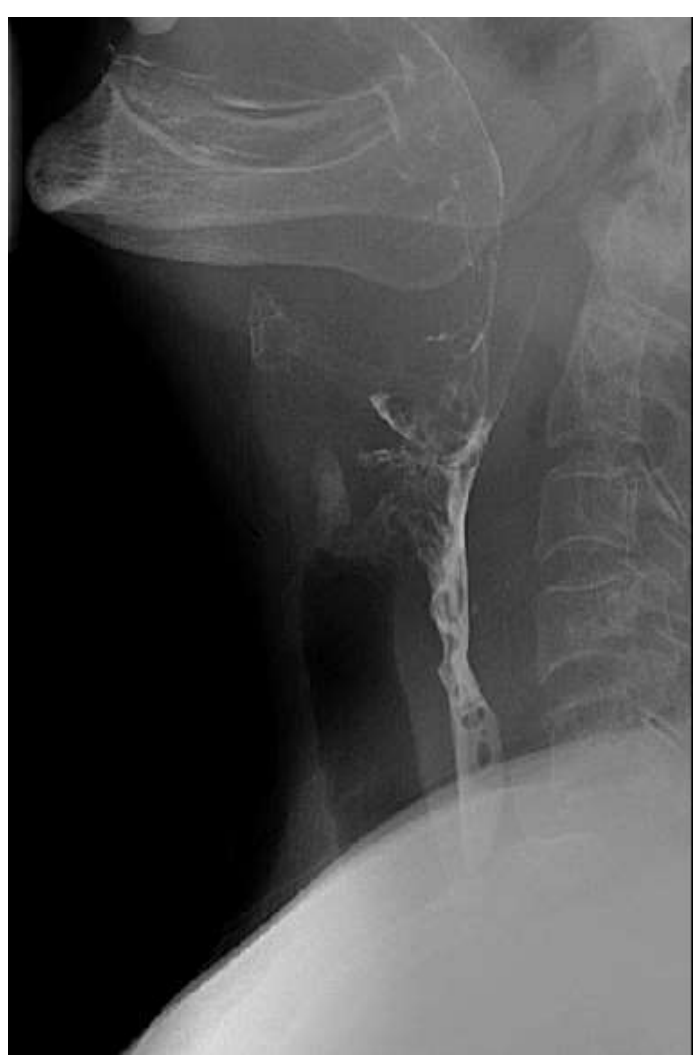

Figure 4: Barium swallow showing resolution of perforation and fistulous tract. 
After 2 days in the ICU, the patient was shifted to a regular ward. Repeat of the gastrograffin study 2 days later showed a fistulous tract from the posterior hypopharyngeal wall at the level of $\mathrm{C} 4$ coursing downwards to the level of $\mathrm{C} 7$. The patient was kept on nasogastric tube and started on nasogastric feeds. The management plan was discussed with the vascular surgery team and it was agreed to treat the patient conservatively with antibiotics, NGT feeds and observation. Repeats of barium swallow tests few days later showed stable appearance of the fistulaous tract. Repeated lateral neck X-rays showed no further complications. The patient was discharged home after 27 days of admission, while still on NG tube. She followed up as an outpatient and the NG tube was removed. It was kept for a total of 40 days.

Repeat of the CT scan and barium swallow done 2 months after the initial presentation showed resolution of the perforation and fistulous tract and the patient's symptoms had resolved.

\section{DISCUSSION}

Fish bone ingestion is a common reason for seeking emergent medical care. As with our patient, fish bone impaction in the esophagus appeared to be more common in older age groups. The reason for this might be due to the physiological characteristics of the esophagus and the deterioration of the swallowing mechanism with advancing age. ${ }^{5}$

Esophageal perforation occurs in about 1-4\% of cases of foreign body ingestion in the esophagus. This can be complicated by death due to suppuration or involvement of major vascular structures. ${ }^{6}$

The management of foreign bodies in the esophagus is a controversial issue. The type of management can be determined by the site of perforation, the general condition of the patient and the degree of contamination. Surgery is considered the mainstay of treatment, however, recent studies suggest a trend more towards nonoperative management. This can include intravenous access and intravenous fluids, nil per oral, nasogastric tube and nasogastric feeds, broad spectrum antibiotics, cardiopulmonary monitoring in the intensive care setting and supplemental oxygen. ${ }^{7}$

This type of treatment can usually be implemented in small and contained esophageal perforations. ${ }^{7,8}$ Patients who develop signs and symptoms of sepsis, or in whom radiologic investigations demonstrate non-contained perforation with contamination should be considered for urgent surgical management. ${ }^{8,9}$

Our patient's condition was monitored by serial plain Xrays, CT scans and gastrograffin studies. Gradual improvement and resolution of the pathology was reassuring to continue with nonoperative management.

\section{CONCLUSION}

Esophageal perforation is a serious complication of foreign body ingestion. If not managed appropriately, it can lead to fistulation, abscess formation and possibly death. Although most cases require surgical management, conservative management is an alternative which can lead to favorable outcomes, especially in patients with comorbidities.

\section{Funding: No funding sources \\ Conflict of interest: None declared \\ Ethical approval: Not required}

\section{REFERENCES}

1. Selivanov V, Sheldon GF, Cello JP, Crass RA. Management of foreign body ingestion. Ann Surg. 1984;199(2):187-91.

2. Venkatesh SH, Venkatanarasimha Karaddi NK. CT findings of accidental fish bone ingestion and its complications. Diagn Interv Radiol. 2016;22(2):156-60.

3. Kim HU. Oroesophageal fish bone foreign body. Clin Endosc. 2016;49(4):318-26.

4. Toma EA, Oun M, Enciu O, Calu V, Miron A. The surgical management of acute esophageal perforation by accidentally ingested fish bone. Chirurgia (Bucur). 2018;113(1):156-61.

5. Kim JP, Kwon OJ, Shim HS, Kim RB, Kim JH, Woo SH. Analysis of clinical feature and management of fish bone ingestion of upper gastrointestinal tract. Clin Exp Otorhinolaryngol. 2015;8(3):261-7.

6. Bathla G, Teo LL, Dhanda S. Pictorial essay: complications of a swallowed fish bone. Indian $\mathrm{J}$ Radiol Imaging. 2011;21(1):63-8.

7. Kaman L, Iqbal J, Kundil B, Kochhar R. Management of Esophageal Perforation in Adults. Gastroenterology Res. 2010;3(6):235-44.

8. Chirica M, Kelly MD, Siboni S, Aiolfi A, Riva GC, Asti E, et al. Esophageal emergencies: WSES guidelines. World J Emerg Surg. 2019;14:26.

9. Altorjay A, Kiss J, Vörös A, Bohák A. Nonoperative management of esophageal perforations. Is it justified? Ann Surg. 1997;225(4):415-21.

Cite this article as: Almarzooq $\mathrm{J}$, Alkhabbaz A, Abdulla N. Management of esophageal perforation with fistula formation in an 80 year old woman with multiple co-morbidities. Int J Otorhinolaryngol Head Neck Surg 2021;7:882-4. 